EUROPEAN JOURNAL OF PURE AND APPLIED MATHEMATICS

Vol. 11, No. 1, 2018, 69-78

ISSN 1307-5543 - www.ejpam.com

Published by New York Business Global

\title{
Application of Probabilistic Method on Daehee Sequences
}

Chang $\mathrm{Liu}^{1, *}$, Wuyungaowa ${ }^{1}$

${ }^{1}$ Department of Mathematical Sciences, Inner Mongolia University, Hohhot, Inner Mongolia, P.R.China

Abstract. In this paper, we investigate some combinatorial sequences based on Daehee and Changhee numbers and polynomials, then derive their moment representations in use of probabilistic method. We also provide identities related to Daehee numbers, derangement numbers, Cauchy numbers of the second kind, and Stirling numbers of the first kind.

2010 Mathematics Subject Classifications: 11B68, 60E07, 11B83, 62E15.

Key Words and Phrases: Moment, Probabilistic method, Generating function, Daehee numbers, Changhee numbers.

\section{Introduction and Preliminaries}

Throughout this paper, we use the following notations:

$$
\mathbb{N}=\{1,2,3, \cdots\}, \mathbb{Z}_{\geqslant 0}=\{0,1,2, \cdots\} .
$$

Let $D_{n, \xi}^{(k)}(x)$ denote the $n$th twisted Daehee polynomials of order $k(\in \mathbb{N})$, which are defined by the generating function[2] to be

$$
\left(\frac{\ln (1+\xi t)}{\xi t}\right)^{k}(1+\xi t)^{x}=\sum_{n=0}^{\infty} D_{n, \xi}^{(k)}(x) \frac{t^{n}}{n !}
$$

In special case, when $x=0, D_{n, \xi}^{(k)}=D_{n, \xi}^{(k)}(0)$ are called twisted Daehee numbers of order $k$. Similarly, $D_{n}^{(k)}=D_{n, 1}^{(k)}$ are higher-order Daehee numbers, $D_{n, \xi}=D_{n, \xi}^{(1)}$ are twisted Daehee numbers, and $D_{n}=D_{n, 1}^{(1)}$ are Daehee numbers.

Let $\hat{D}_{n, \xi}^{(k)}(x)$ denote the $n$th twisted Daehee polynomials of the second kind of order $k(\in \mathbb{N})$, which are defined by the generating function[2] to be

${ }^{*}$ Corresponding author.

Email addresses: chang12013@hotmail.com (C. Liu), wuyungw@163.com (Wuyungaowa) 


$$
\left(\frac{(1+\xi t) \ln (1+\xi t)}{\xi t}\right)^{k}(1+\xi t)^{x}=\sum_{n=0}^{\infty} \hat{D}_{n, \xi}^{(k)}(x) \frac{t^{n}}{n !} .
$$

In special case, when $x=0, \hat{D}_{n, \xi}^{(k)}=\hat{D}_{n, \xi}^{(k)}(0)$ are called higher-order twisted Daehee numbers of the second kind. Similarly, $\hat{D}_{n}^{(k)}=\hat{D}_{n, 1}^{(k)}$ are higher-order Daehee numbers of the second kind, $\hat{D}_{n, \xi}=\hat{D}_{n, \xi}^{(1)}$ are twisted Daehee numbers of the second kind, and $\hat{D}_{n}=$ $\hat{D}_{n, 1}^{(1)}$ are Daehee numbers of the second kind.

Let $C h_{n}^{(r)}(x)$ denote the $n$th Changhee polynomials of order $r(\in \mathbb{N})$, which are defined by the generating function[1] to be

$$
\left(\frac{2}{2+t}\right)^{r}(1+t)^{x}=\sum_{n=0}^{\infty} C h_{n}^{(r)}(x) \frac{t^{n}}{n !} .
$$

When $x=0, C h_{n}^{(r)}(0)=C h_{n}^{(r)}$ are called higher-order Changhee numbers.

Let $\hat{C} h_{n}^{(r)}(x)$ denote the $n$th Changhee polynomials of order $r(\in \mathbb{N})$ of the second kind, which are defined by the generating function[1] to be

$$
\left(\frac{2}{2+t}\right)^{r}(1+t)^{x+r}=\sum_{n=0}^{\infty} \hat{C} h_{n}^{(r)}(x) \frac{t^{n}}{n !} .
$$

When $x=0, \hat{C} h_{n}^{(r)}(0)=\hat{C} h_{n}^{(r)}$ are called higher-order Changhee numbers of the second kind.

Remark 1. [see 7] If $f$ and $g$ are exponential generating functions, and

$$
f g=\left(\sum_{r=0}^{\infty} \frac{a_{r} x^{r}}{r !}\right)\left(\sum_{s=0}^{\infty} \frac{b_{s} x^{s}}{s !}\right)
$$

then the coefficients of $\frac{x^{n}}{n !}$ in $f g$ are given by

$$
\left[\frac{x^{n}}{n !}\right](f g)=\sum_{r=0}^{n}\left(\begin{array}{l}
n \\
r
\end{array}\right) a_{r} b_{n-r} .
$$

Remark 2. Throughout this paper, symbol E denotes the expectation operator defined by

$$
E f(X)=\int_{-\infty}^{+\infty} f(x) p(x) d x
$$

where random variable $X$ is continuous, whose density function is $p(x)$. Specially, when $f(x)=x^{n}, E X^{n}$ denotes $n$-order moment of random variable $X$.

(i) When r.v $u \sim U[0,1], E u^{n}=\frac{1}{n+1}$, 
(ii) When r.v $X \sim \Gamma(1,1), E X^{n}=n$ !.

Definition 1. The characteristic function of random variable $X$ is defined as

$$
\varphi(t)=E e^{i t X}, i^{2}=-1,-\infty<t<\infty .
$$

When the moments of all orders of r.v. $X$ exist, the following relation expression holds true,

$$
E X^{n}=\left[\frac{(i t)^{n}}{n !}\right] \varphi(t), \quad i^{2}=-1 .
$$

Remark 3. [see 5] If random variable $X$ is distributed as $\Gamma(\alpha, \lambda)$, where $\alpha, \lambda>0$, its characteristic function is

$$
\varphi(t)=E e^{i t X}=\left(1-\frac{i t}{\lambda}\right)^{-\alpha} .
$$

Remark 4. $X$ and $Y$ are two random variables, when $\operatorname{Cov}(X, Y)=0$, we have $E(X Y)=E X \cdot E Y$, where

$$
\operatorname{Cov}(X, Y)=E(X Y)-E(X) E(Y)
$$

Then we give three lemmas to introduce moment representations of some special combinatorial sequences.

Lemma 1. [see 6] Assume that $r . v X \sim \Gamma(u, 1)$, with $u \sim U[0,1]$ is a random variable that follows uniform distribution, and $X, u$ are independent respectively, then Cauchy numbers of the second kind $\hat{C}_{n}$, whose generating function is

$$
\sum_{n=0}^{\infty} \hat{C}_{n} \frac{x^{n}}{n !}=\frac{x}{(1+x) \ln (1+x)},
$$

have the following moment representation,

$$
\hat{C}_{n}=E X^{n}, \quad n \geqslant 0 .
$$

Lemma 2. [see 6] Assume that r.v $X \sim \Gamma(1,1)$, then derangement numbers $d_{n}=$ $n ! \sum_{k=0}^{n} \frac{(-1)^{k}}{k !}$ have the following moment representation,

$$
d_{n}=E(X-1)^{n}, \quad n \geqslant 0 .
$$

Lemma 3. [see 6] Suppose that r.v.s $u_{1}, u_{2}, \cdots$, i.i.d $\sim U[0,1]$, r.v.s $\Gamma_{1}, \Gamma_{2}, \cdots$, i.i.d $\Gamma(1,1)$, r.v $u_{i}$ and $\Gamma_{j}$ are independent respectively for all $i, j$. When $n, k \geq 1$, Stirling numbers of the first kind $s(n, k)$ satisfy

$$
s(n, k)=(-1)^{n-k}\left(\begin{array}{l}
n \\
k
\end{array}\right) E\left(u_{1} \Gamma_{1}+u_{2} \Gamma_{2}+\cdots+u_{k} \Gamma_{k}\right)^{n-k} .
$$

It is demanded that $s(n, 0)=s(0, k)=0, s(0,0)=1$. 
C. Liu, Wuyungaowa / Eur. J. Pure Appl. Math, 11 (1) (2018), 69-78

\section{Moment Representations of Daehee and Changhee Sequences}

In this section, we use probabilistic method to derive moment representations about two kinds of higher-order twisted Daehee numbers and polynomials, and two kinds of higher-order Changhee numbers and polynomials.

Theorem 1. Assume that r.v.s $u_{1}, u_{2}, \ldots$, i.i.d $\sim U[0,1], \Gamma_{1}, \Gamma_{2}, \ldots, i . i . d \sim \Gamma(1,1)$, and for all $i, j$, r.v $u_{i}$ and $\Gamma_{j}$ are independent. When $n, m-k \in \mathbb{Z}_{\geqslant 0}, k \in \mathbb{N}$, we have

$$
\begin{gathered}
D_{n, \xi}^{(k)}=(-\xi)^{n} E\left(u_{1} \Gamma_{1}+u_{2} \Gamma_{2}+\cdots+u_{k} \Gamma_{k}\right)^{n}, \\
D_{m-k, \xi}^{(k)}=(-\xi)^{m-k} \frac{k}{m} E\left(u_{1} \Gamma_{1}+\cdots+u_{k-1} \Gamma_{k-1}+\Gamma_{k}\right)^{m-k} .
\end{gathered}
$$

Proof. The generating function of higher-order twisted Daehee numbers is known as

$$
\sum_{n=0}^{\infty} D_{n, \xi}^{(k)} \frac{t^{n}}{n !}=\left(\frac{\ln (1+\xi t)}{\xi t}\right)^{k},
$$

Taking the coefficients of $t^{n}$ in the left-hand side of Eq.(14), we get

$$
\begin{aligned}
\frac{D_{n, \xi}^{(k)}}{n !} & =\left[t^{n}\right]\left(\frac{\ln (1+\xi t)}{\xi t}\right)^{k}=\left[(-t)^{n}\right]\left(\sum_{i \geqslant 0} \frac{(\xi t)^{i}}{i+1}\right)^{k}=\left[(-t)^{n}\right]\left(\sum_{i \geqslant 0} t^{i} E(\xi u)^{i}\right)^{k}, \\
\left(\sum_{i \geqslant 0} t^{i} E(\xi u)^{i}\right)^{k} & =\sum_{n=0}^{\infty} \sum_{i_{1}+\cdots+i_{k}=n}\left(E\left(\xi u_{1}\right)^{i_{1}}\right) \cdots\left(E\left(\xi u_{k}\right)^{i_{k}}\right) t^{n} \\
& =\sum_{n=0}^{\infty} \frac{1}{n !} \sum_{i_{1}+\cdots+i_{k}=n}\left(\begin{array}{c}
n \\
i_{1}, i_{2}, \cdots i_{k}
\end{array}\right)\left(E\left(\xi u_{1}\right)^{i_{1}}\right) \cdots\left(E\left(\xi u_{k}\right)^{i_{k}}\right)\left(i_{1} !\right) \cdots\left(i_{k} !\right) t^{n} \\
& =\sum_{n=0}^{\infty} \frac{1}{n !} \sum_{i_{1}+\cdots+i_{k}=n}\left(\begin{array}{c}
n \\
i_{1}, i_{2}, \cdots i_{k}
\end{array}\right)\left(E\left(\xi u_{1}\right)^{i_{1}}\right) \cdots\left(E\left(\xi u_{k}\right)^{i_{k}}\right)\left(E \Gamma_{1}^{i_{1}}\right) \cdots\left(E \Gamma_{k}^{i_{k}}\right) t^{n} \\
& =\sum_{n=0}^{\infty} \frac{\xi^{n}}{n !} E\left[\sum_{i_{1}+\cdots+i_{k}=n}\left(\begin{array}{c}
n \\
i_{1}, i_{2}, \cdots i_{k}
\end{array}\right)\left(u_{1} \Gamma_{1}\right)^{i_{1}} \cdots\left(u_{k} \Gamma_{k}\right)^{i_{k}}\right] t^{n} \\
& =\sum_{n=0}^{\infty} \frac{\xi^{n}}{n !} E\left(u_{1} \Gamma_{1}+\cdots+u_{k} \Gamma_{k}\right)^{n} t^{n} .
\end{aligned}
$$

From Eq.(15) and Eq.(16), we can see that

$$
\frac{D_{n, \xi}^{(k)}}{n !}=\frac{(-\xi)^{n}}{n !} E\left(u_{1} \Gamma_{1}+\cdots+u_{k} \Gamma_{k}\right)^{n},
$$

thus we have

$$
D_{n, \xi}^{(k)}=(-\xi)^{n} E\left(u_{1} \Gamma_{1}+\cdots+u_{k} \Gamma_{k}\right)^{n} .
$$


C. Liu, Wuyungaowa / Eur. J. Pure Appl. Math, 11 (1) (2018), 69-78

Eq.(13) can be proved by the following equation[see 6]

$$
E\left(u_{1} \Gamma_{1}+u_{2} \Gamma_{2}+\cdots+u_{k} \Gamma_{k}\right)^{m-k}=\frac{k}{m} E\left(u_{1} \Gamma_{1}+\cdots+u_{k-1} \Gamma_{k-1}+\Gamma_{k}\right)^{m-k}
$$

Corollary 1. In theorem 1, when $\xi=1$, we obtain the moment representation of higherorder Daehee numbers[3]

$$
D_{n}^{(k)}=(-1)^{n} E\left(u_{1} \Gamma_{1}+\cdots+u_{k} \Gamma_{k}\right)^{n}
$$

Corollary 2. In theorem 1, when $k=1$, we obtain the moment representation of twisted Daehee numbers[2]

$$
D_{n, \xi}=(-\xi)^{n} E(u \Gamma)^{n}
$$

Corollary 3. In theorem1, when $\xi=1, k=1$, we obtain the moment representation of Daehee numbers[4]

$$
D_{n}=(-1)^{n} E(u \Gamma)^{n} \text {. }
$$

Theorem 2. Suppose that r.v $u \sim U[0,1], \Gamma \sim \Gamma(1,1), r . v u$ and $\Gamma$ are independent, then twisted Daehee numbers of the second kind of order $k$ satisfy

$$
\hat{D}_{n, \xi}^{(k)}=\sum_{i=0}^{n}\left(\begin{array}{l}
n \\
i
\end{array}\right) \xi^{n-i}(k)_{n-i} D_{i, \xi}^{(k)}
$$

Proof. The generating function of $\hat{D}_{n, \xi}^{(k)}$ is given by

$$
\left(\frac{\ln (1+\xi t)}{\xi t}(1+\xi t)\right)^{k}=\sum_{n=0}^{\infty} \hat{D}_{n, \xi}^{(k)} \frac{t^{n}}{n !}
$$

From theorem 1, the left-hand side of Eq.(22) can be written as

$$
\begin{aligned}
& \left(\frac{\ln (1+\xi t)}{\xi t}\right)^{k}(1+\xi t)^{k} \\
& =\sum_{n=0}^{\infty}(-\xi)^{n} E\left(u_{1} \Gamma_{1}+u_{2} \Gamma_{2}+\cdots+u_{k} \Gamma_{k}\right)^{n} \frac{t^{n}}{n !} \sum_{n=0}^{\infty}(k)_{n} \frac{(\xi t)^{n}}{n !} \\
& =\sum_{n=0}^{\infty} \sum_{i=0}^{n}\left(\begin{array}{l}
n \\
i
\end{array}\right)(-\xi)^{i} E\left(u_{1} \Gamma_{1}+u_{2} \Gamma_{2}+\cdots+u_{k} \Gamma_{k}\right)^{i}(k)_{n-i} \xi^{n-i} \frac{t^{n}}{n !} \\
& =\sum_{n=0}^{\infty} \sum_{i=0}^{n}\left(\begin{array}{l}
n \\
i
\end{array}\right) \xi^{n-i}(k)_{n-i} D_{i, \xi}^{(k)} \frac{t^{n}}{n !},
\end{aligned}
$$

where $(k)_{n}=k(k-1) \cdots(k-n+1)$. By comparing the coefficients of $\frac{t^{n}}{n !}$, theorem 2 is proved. 
Corollary 4. In theorem 2, taking $k=1, n \geqslant 1$, we obtain the moment form of twisted Daehee numbers of the second kind,

$$
\hat{D}_{n, \xi}=\frac{(-1)^{n-1}}{n} \xi^{n} E(u \Gamma)^{n}, n \geqslant 1, \hat{D}_{0, \xi}=1 .
$$

Corollary 5. From corollary 2 and corollary 4, when $n \geqslant 1$, the following relationship holds true,

$$
-n \hat{D}_{n, \xi}=D_{n, \xi}, \quad n \geqslant 1 \text {. }
$$

Theorem 3. Under the circumstance of theorem 1, higher-order twisted Daehee polynomials $D_{n, \xi}^{(k)}(x)$ have the following moment representation3

$$
D_{n, \xi}^{(k)}(x)=\sum_{i=0}^{n}\left(\begin{array}{c}
n \\
i
\end{array}\right) \xi^{n-i}(x)_{n-i} D_{i, \xi}^{(k)}
$$

Proof. Proof of theorem 3 is similar to the one of theorem 2.

Theorem 4. Suppose that r.v.s $u_{1}, u_{2}, \ldots$, i.i.d $\sim U[0,1], \Gamma_{1}, \Gamma_{2}, \ldots$, i.i.d $\sim \Gamma(1,1)$, $X \sim \Gamma\left[-x, \frac{1}{\xi}\right], \quad(x<0, \xi>0)$, and $r . v u_{i}, \Gamma_{j}$ and $X$ are independent for all $i, j$, then higher-order twisted Daehee polynomials $D_{n, \xi}^{(k)}(x)$ satisfy

$$
D_{n, \xi}^{(k)}(x)=E\left[\xi\left(u_{1} \Gamma_{1}+\cdots+u_{k} \Gamma_{k}\right)+X\right]^{n} .
$$

Proof. Replacing $t$ by -it in the generating function of higher-order twisted Daehee polynomials, and according to remark 3 and 4 , we have

$$
\begin{aligned}
\sum_{n=0}^{\infty} D_{n, \xi}^{(k)}(x) \frac{(-i t)^{n}}{n !} & =\left(\frac{\ln (1-\xi i t)}{-\xi i t}\right)^{k}(1-\xi i t)^{-(-x)} \\
& =\sum_{n=0}^{\infty}(-1)^{n} \xi^{n} E\left(u_{1} \Gamma_{1}+\cdots+u_{k} \Gamma_{k}\right)^{n} \frac{(-i t)^{n}}{n !} \sum_{n=0}^{\infty} E X^{n} \frac{(i t)^{n}}{n !} \\
& =\sum_{n=0}^{\infty} \sum_{i=0}^{n}\left(\begin{array}{c}
n \\
i
\end{array}\right) \xi^{i} E\left(u_{1} \Gamma_{1}+\cdots+u_{k} \Gamma_{k}\right)^{i} E X^{n-i} \frac{(i t)^{n}}{n !} \\
& =\sum_{n=0}^{\infty} E\left[\xi\left(u_{1} \Gamma_{1}+\cdots+u_{k} \Gamma_{k}\right)+X\right]^{n} \frac{i t^{n}}{n !}
\end{aligned}
$$

By comparing the coefficients of $\frac{(i t)^{n}}{n !}$, we obtain theorem 4 .

Theorem 5. Suppose that r.v $u \sim U[0,1], \Gamma \sim \Gamma(1,1), r \cdot v u$ and $\Gamma$ are independent respectively, twisted Daehee polynomials of the second kind of order $k$ satisfy

$$
\hat{D}_{n, \xi}^{(k)}(x)=\sum_{i=0}^{n}\left(\begin{array}{c}
n \\
i
\end{array}\right) \xi^{n}(-1)^{i} E\left(u_{1} \Gamma_{1}+\cdots+u_{k} \Gamma_{k}\right)^{i}(x+k)_{n-i} .
$$


Proof. Proof of theorem 5 is similar to that of theorem 2.

Theorem 6. Suppose that r.v.s $u_{1}, u_{2}, \ldots$, i.i.d $\sim U[0,1], \Gamma_{1}, \Gamma_{2}, \ldots$, i.i.d $\sim \Gamma(1,1)$, $X \sim \Gamma\left[-x-k, \frac{1}{\xi}\right],(x<-k, \xi>0)$, and r.v $u_{i}, \Gamma_{j}$ and $X$ are independent for all $i, j$, then higher-order twisted Daehee polynomials of the second kind $\hat{D}_{n, \xi}^{(k)}(x)$ satisfy

$$
\hat{D}_{n, \xi}^{(k)}(x)=(-1)^{n} E\left[\xi\left(u_{1} \Gamma_{1}+\cdots+u_{k} \Gamma_{k}\right)+X\right]^{n} .
$$

Proof. Proof of theorem 6 is similar to the one of theorem 4.

Theorem 7. Assume r.v $X \sim \Gamma[r, 2]$, then higher-order Changhee numbers satisfy

$$
C h_{n}^{(r)}=(-1)^{n} E X^{n} \text {. }
$$

Proof. Replacing $t$ by $-i t$, where $i^{2}=-1$ in the generating function of $C h_{n}^{(r)}$, we have

$$
\left(\frac{2}{2-i t}\right)^{r}=\sum_{n=0}^{\infty} C h_{n}^{(r)} \frac{(-i t)^{n}}{n !}
$$

The left-hand side of Eq.(30) can be written as

$$
\left(1-\frac{i t}{2}\right)^{-r}=E e^{i t X}=\sum_{n=0}^{\infty} E X^{n} \frac{(i t)^{n}}{n !},
$$

from Eq.(30) and Eq.(31), we obtain

$$
\sum_{n=0}^{\infty} C h_{n}^{(r)} \frac{(-i t)^{n}}{n !}=\sum_{n=0}^{\infty}(-1)^{n} E X^{n} \frac{(-i t)^{n}}{n !} .
$$

By comparing the coefficients of $\frac{(-i t)^{n}}{n !}$, theorem 7 is proved.

Theorem 8. Under the circumstance of theorem 7, higher-order Changhee polynomials of the second kind $\hat{C} h_{n}^{(r)}(x)$ satisfy

$$
\hat{C} h_{n}^{(r)}(x)=\sum_{k=0}^{n}\left(\begin{array}{l}
n \\
k
\end{array}\right)(-1)^{k}(x+r)_{n-k} E X^{k} .
$$

Proof. The generating function of $\hat{C} h_{n}^{(r)}(x)$ can be written as

$$
\begin{aligned}
\sum_{n=0}^{\infty} \hat{C} h_{n}^{(r)}(x) \frac{t^{n}}{n !} & =\left(\frac{2}{2+t}\right)^{r}(1+t)^{x+r} \\
& =\sum_{n=0}^{\infty}(-1)^{n} E X^{n} \frac{t^{n}}{n !} \sum_{n=0}^{\infty}(x+r)_{n} \frac{t^{n}}{n !}
\end{aligned}
$$




$$
=\sum_{n=0}^{\infty} \sum_{k=0}^{n}\left(\begin{array}{l}
n \\
k
\end{array}\right)(-1)^{k} E X^{k}(x+r)_{n-k} \frac{t^{n}}{n !} .
$$

By comparing the coefficients of $\frac{t^{n}}{n !}$, theorem 8 is proved.

Corollary 6. In theorem 8 , taking $x=0$, we obtain the moment form of higher-order Changhee numbers of the second kind,

$$
\hat{C} h_{n}^{(r)}=\sum_{k=0}^{n}\left(\begin{array}{l}
n \\
k
\end{array}\right)(-1)^{k}(r)_{n-k} E X^{k} .
$$

Corollary 7. Since the generating function of higher-order Changhee polynomials is

$$
\sum_{n=0}^{\infty} C h_{n}^{(r)}(x) \frac{t^{n}}{n !}=\left(\frac{2}{t+2}\right)^{r}(1+t)^{x} .
$$

From theorem 8, we can see that the moment form of $C h_{n}^{(r)}(x)$ is

$$
C h_{n}^{(r)}(x)=\sum_{k=0}^{n}\left(\begin{array}{l}
n \\
k
\end{array}\right)(-1)^{k}(x)_{n-k} E X^{k} .
$$

\section{Identities of Daehee Numbers and Special Combinatorial Sequences}

In this section, we use moment forms of special combinatorial sequences, characteristic function and generating function method to investigate the relationships between Daehee numbers $D_{n}$, Cauchy numbers of the second kind $\hat{C}_{n}$, derangement numbers $d_{n}$, and Stirling numbers of the first kind, then we obtain combinatorial identities about them.

Theorem 9. Let r.v $u \sim U[0,1], \Gamma \sim \Gamma(1,1), X \sim \Gamma(u, 1)$, then Daehee numbers $D_{n}$, Cauchy numbers of the second kind $\hat{C}_{n}$, and derangement numbers $d_{n}$ satisfy the following identity

$$
\sum_{k=0}^{n}\left(\begin{array}{l}
n \\
k
\end{array}\right)(-1)^{n} D_{k} \hat{C}_{n-k}=\sum_{k=0}^{n}\left(\begin{array}{l}
n \\
k
\end{array}\right) d_{k}, n \geqslant 0 .
$$

Proof. On one hand,

$$
\begin{aligned}
{[E(u \Gamma)-E X]^{n} } & =\sum_{k=0}^{n}\left(\begin{array}{l}
n \\
k
\end{array}\right) E(u \Gamma)^{k}(-E X)^{n-k} \\
& =\sum_{k=0}^{n}\left(\begin{array}{l}
n \\
k
\end{array}\right)(-1)^{n} E(-u \Gamma)^{k} E X^{n-k}
\end{aligned}
$$


C. Liu, Wuyungaowa / Eur. J. Pure Appl. Math, 11 (1) (2018), 69-78

$$
=\sum_{k=0}^{n}\left(\begin{array}{l}
n \\
k
\end{array}\right)(-1)^{n} D_{k} \hat{C}_{n-k}
$$

Write the generating function of the equation above, noting that $i^{2}=-1$

$$
\begin{aligned}
\sum_{n=0}^{\infty}[E(u \Gamma)-E X]^{n} \frac{(i t)^{n}}{n !} & =\sum_{n=0}^{\infty} \sum_{k=0}^{n}\left(\begin{array}{l}
n \\
k
\end{array}\right)(-1)^{n} D_{k} \hat{C}_{n-k} \frac{(i t)^{n}}{n !} \\
& =\sum_{n=0}^{\infty} D_{n} \frac{(-i t)^{n}}{n !} \sum_{n=0}^{\infty} \hat{C}_{n} \frac{(-i t)^{n}}{n !} \\
& =\frac{\ln (1-i t)}{-i t} \frac{-i t}{(1-i t) \ln (1-i t)} \\
& =E e^{i t \Gamma}=\sum_{n=0}^{\infty} E \Gamma^{n} \frac{(i t)^{n}}{n !}
\end{aligned}
$$

On the other hand, by comparing the coefficients of $\frac{(i t)^{n}}{n !}$ in Eq.(38), we have

$$
[E(u \Gamma)-E X]^{n}=E \Gamma^{n}=E(\Gamma-1+1)^{n}=\sum_{k=0}^{n}\left(\begin{array}{l}
n \\
k
\end{array}\right) E(\Gamma-1)^{k}=\sum_{k=0}^{n}\left(\begin{array}{l}
n \\
k
\end{array}\right) d_{k} .
$$

From Eq.(37) and Eq.(39), we can get Eq.(36). Theorem 9 is proved.

Theorem 10. Higher-order Deahee numbers $D_{n}^{(k)}$ and Stirling numbers of the first kind satisfy the following relationship

$$
D_{n}^{(k)}=\frac{s(n+k, k)}{\left(\begin{array}{c}
n+k \\
k
\end{array}\right)} .
$$

Proof. From corollary 1 and lemma 3, write generating function of the left-hand side of Eq.(40),

$$
\begin{aligned}
\sum_{n=0}^{\infty} D_{n}^{(k)} \frac{t^{n}}{n !} & =\sum_{n=0}^{\infty}(-1)^{n} E\left(u_{1} \Gamma_{1}+\cdots+u_{k} \Gamma_{k}\right)^{n} \frac{t^{n}}{n !} \\
& =\sum_{n=0}^{\infty}(-1)^{n}\left(\begin{array}{c}
n+k \\
k
\end{array}\right) E\left(u_{1} \Gamma_{1}+\cdots+u_{k} \Gamma_{k}\right)^{n} \frac{k !}{t^{k}} \frac{t^{n+k}}{(n+k) !} \\
& =\sum_{n=0}^{\infty} \frac{s(n+k, k)}{\left(\begin{array}{c}
n+k \\
k
\end{array}\right)} \frac{t^{n}}{n !} .
\end{aligned}
$$

By comparing the coefficients of $\frac{t^{n}}{n !}$, we obtain the conclusion. 


\section{Acknowledgements}

The research is supported by the Natural Science Foundation of China under Grant 11461050 and Natural Science Foundation of Inner Mongolia under Grant 2016MS0104

\section{References}

[1] JJ Seo DS Kim, T Kim and SH Lee. Higher-Order Changhee Numbers and Polynomials. Adv. Studies Theor. Phys., 8(8):365-373, 2014.

[2] SH Lee DS Kim, T Kim and JJ Seo. A Note on the Twisted lambda-Daehee Polynomials. Applied Mathmetical Sciences, 7(141):7005-7014, 2013.

[3] SH Lee DS Kim, T Kim and JJ Seo. Higher-Order Daehee Numbers and Polynomials. Int. Journal of Math. Analysis, 8(6):273-283, 2014.

[4] DS Kim and T Kim. Daehee Numbers and Polynomials. Applied Mathmetical Sciences, 7(120):5969-5976, 2013.

[5] E Lukacs. Chracteristic Function. Charles Griffin and Company Limited, London, 1960.

[6] P Sun and T Wang. Probabilistic Representation with Application of Stirling Numbers. Acta Mathematica Sinica Chinese Series, 41(2):281-290, 1998.

[7] HS Wilf. Generating Functionology. Academic Press, New York, 1990. 